\title{
Time to relax the rules about administration of mifepristone
}

\author{
Salmon Omokanye, FMCOG, MRCOG, MFFP, Senior Clinical Medical Officer
}

Correspondence. Dr S Omokanye, SCMO, Family Planning and Reproductive Health Care, Nottingham Community Health NHS Trust, Victoria Health Centre, Glass House Street, Nottingham, NG1 3LW, UK.

(Accepted January $20^{\text {th }}, 2001$ )

\begin{abstract}
Summary
Pre-clinical and clinical data indicate that mifepristone is a safe and effective drug for the termination of pregnancy. The author suggests that it could be given by nursing staff rather than by a doctor, and that the 2-hour postadministration observation period is unnecessary in the majority of cases.
\end{abstract}

\section{Key words}

abortion, mifepristone

Mifepristone is effective and safe for early pregnancy termination..$^{1-3}$

The manufacturer's data sheet and control procedures in hospitals include the following rules, which often create practical problems:

1. administration of oral mifepristone in the presence of the doctor

2. the patient should be observed for at least 2 hours following administration.

There is some variation in the extent to which service providers respect these rules. Some centres have no problem making a doctor available to observe the ingestion of the tablets. In small units, strict adherence to the rules means that some patients have to bear waiting for the doctor.

In our experience at Bassetlaw Hospital, Worksop, with medical termination of pregnancy for 40 to 50 patients per year (1993 - 1998), there were only four patients on our record who reported transient nausea, and only one patient actually vomited. We observed no other adverse effect. All the patients felt well and ready to go home as soon as possible, but we kept them under observation for 2 hours in compliance with our protocol.

The most remarkable pharmacodynamic of mifepristone is the antiglucocorticoid effect for a minimum of 24 hours after a single oral administration. However, no clinical or biological signs of adrenal insufficiency have ever been observed. ${ }^{4}$

Faintness and vomiting were reported in clinical trials in $5.1 \%$ and $2.3 \%$ of patients, respectively, but it was difficult to differentiate these symptoms from the effect of the pregnancy and also from the process of abortion. ${ }^{5}$

Toxicological studies in rodents estimated the LD50 to be greater than $1 \mathrm{~g} / \mathrm{kg}^{6}{ }^{6}$ By deduction, the therapeutic ratio for human would be about 1:100 for a $600 \mathrm{mg}$ dose, and 1:200 for the $300 \mathrm{mg}$ dose, which implies that it is safer than paracetamol.

It would therefore be safe and more practical if the prescribing doctor might delegate the supervision of oral administration of mifepristone to nursing staff who also have extended role to administer prostaglandins for termination of pregnancy. ${ }^{7}$ Furthermore, patients who have no relative contraindications, and those who experienced no side effects, may be free to take their leave at once.

\footnotetext{
Statements on funding and competing interests

Funding. None declared.

Competing interests. None.

References

Cameron IT, Michie AF, Baird DT. Therapeutic abortion in early pregnancy with antiprogestogen RU486 alone or in combination with prostaglandin analogue (gemeprost) Contraception 1986; 34: 459-468.

2 Rodger MW, Baird DT. Induction of therapeutic abortion in early pregnancy with mifepristone in combination with prostaglandin pessary. Lancet 1987; ii: $1415-1418$.

UK Multicentre Trial. The efficacy and tolerance of mifepristone and prostaglandin in first trimester termination of pregnancy. Br J Obstetrics and Gynaecology 1990; 97: 480-486.

Gaillard RC, Riondell A, Muller AF, et al. RU486:A steroid with antiglucocorticoid activity that only disinhibits the human pituitary-adrenal system at a specific time of day. Proceedings of The only disinhibits the human pituitary-adrenal system at a specif

Rousell Company Mifegyne Data File. Medical termination of early pregnancy. Rousell Laboratory Publication. Ref.MF6; June 1993.

Deraedt R, Vannier B, Fournex R. Toxicology study on RU486. In: Baulieu EE, Segal SJ (Eds.) The antiprogestin steroid RU486 and human fertility control. New York: Plenum Press, 1985, The antiprogest $123-126$.

pp 123-126.

Department of Health and UK. Central Council. Termination of pregnancy by medical methods: The role of the registered nurse or midwife and others who are not medical practitioners PLCMO (94) 8; PLNCO (94) 10 .
} 